\title{
Essais
}

Revue interdisciplinaire d'Humanités

\section{La presse contre les élites? Le cas de Charlie Hebdo dans les années 1970}

\section{Stéphane Mazurier}

\section{(2) OpenEdition}

1 Journals

Édition électronique

URL : http://journals.openedition.org/essais/8198

DOI : $10.4000 /$ essais. 8198

ISSN : 2276-0970

Éditeur

École doctorale Montaigne Humanités

\section{Édition imprimée}

Date de publication : 15 juillet 2014

Pagination : 68-78

ISBN : 978-2-9544269-3-8

ISSN : 2417-4211

\section{Référence électronique}

Stéphane Mazurier, «La presse contre les élites? Le cas de Charlie Hebdo dans les années 1970 », Essais [En ligne], 5 | 2014, mis en ligne le 13 avril 2021, consulté le 15 avril 2021. URL : http:// journals.openedition.org/essais/8198; DOI : https://doi.org/10.4000/essais.8198 


\section{La presse contre les élites? Le cas de Charlie Hebdo dans les années 1970}

\section{Stéphane Mazurier}

Voici un peu plus de trente ans, en janvier 1982, disparaissait, faute de lecteurs, la première série du journal Charlie Hebdo, initialement appelé HaraKiri Hebdo, lors de sa création en février 1969, puis L'Hebdo Hara-Kiri dès le mois de mai suivant. Sa parution correspond ainsi peu ou prou à la période de "l'entre-deux-mai ", pour reprendre l'heureuse formule de Pascal Ory ${ }^{1}$, située entre Mai 68 et mai 1981, c'est-à-dire l'élection de François Mitterrand à la présidence de la République. Charlie Hebdo est un journal dirigé essentiellement par deux hommes: Georges Bernier, alias le Professeur Choron, qui en est le directeur de publication et l'éditeur, et François Cavanna, son rédacteur en chef. Les deux hommes se sont rencontrés au milieu des années cinquante, dans les bureaux d'un journal nommé Zéro, distribué uniquement par colportage. Bernier en était le directeur des ventes et Cavanna, déjà, le rédacteur en chef. Désireux de fonder leur propre publication, ils ont créé, en compagnie du dessinateur Fred $^{2}$, en septembre 1960, un mensuel, Hara-Kiri, journal bête et méchant, qui, au moyen de dessins, de textes et de photographies, déploie un humour noir et volontiers provocateur ${ }^{3}$. Autour de Cavanna et de Choron se sont réunis de nombreux dessinateurs, dont la plupart ont fait leurs premières armes dans Hara-Kiri : Reiser, Cabu, Wolinski, Gébé, Willem, Fournier... Autre grand nom du dessin d'humour, Siné ne rejoint Charlie Hebdo qu'en 1974, de façon éphémère, puis définitivement à partir de 1980. À la différence des autres dessinateurs, il n'a pas été révélé par Hara-Kiri, mais par sa célèbre

1 Ory Pascal, L'Entre-deux-Mai. Histoire culturelle de la France, mai 1968-mai 1981, Paris, Seuil, 1983.

2 De son vrai nom Frédéric Othon Aristidès, Fred est le directeur artistique de Hara-Kiri, dont il signe le dessin de couverture jusqu'en 1963. Il rejoint Pilote en 1965 et y crée son plus fameux personnage, Philémon.

3 Né dix ans avant Charlie Hebdo, le mensuel Hara-Kiri disparaît des kiosques quelques années après son cadet, à la fin 1985. Dans Bête et méchant (Paris, Belfond, 1981), Cavanna se livre à une définition précise du type d'humour pratiqué dans Hara-Kiri. 
série des célèbres " chats » à partir de la fin des années cinquante ${ }^{4}$, avant de participer à L'Express, de 1958 à 1962, puis de fonder ses propres journaux : Siné massacre et L'Enragé.

Charlie Hebdo n'est cependant pas qu'un journal de dessinateurs : outre les articles de Cavanna, on peut y lire des textes de Delfeil de Ton, d'Isabelle Cabut, puis, plus tard, de Jackie Berroyer, Jean-Patrick Manchette ou encore Sylvie Caster.

Journal à part dans le paysage médiatique français des années 1970, Charlie Hebdo cultive ainsi un rapport complexe avec les élites de son temps. Il peut, en effet, adopter une posture farouchement hostile aux classes dominantes, qu'il s'agisse du patronat, des responsables politiques du gouvernement et de la majorité de droite, ou bien encore de certains magnats de la presse. En revanche, Charlie Hebdo s'intègre progressivement à une sorte d'élite alternative ou de " contre-élite ", en tant que journal d'une certaine avant-garde artistique, mais aussi en raison d'une participation accrue de plusieurs de ses membres à des réseaux du pouvoir politique et culturel.

\section{Charlie Hebdo et les élites : 1'histoire d'un affrontement?}

Dans la grande tradition de la presse satirique française, comme L'Assiette au beurre (1901-1912), Charlie Hebdo concentre ses attaques sur les dépositaires du pouvoir, ou plutôt des pouvoirs. Ainsi, le journal, notamment par le biais de ses dessinateurs, adopte un ton très sarcastique à l'endroit des élites entrepreneuriales, sans jamais toutefois basculer dans la violence idéologique ou dans l'appel au meurtre que pouvait alors lancer la presse d'extrême gauche 5 . On s'amuse simplement des vagues de séquestration que subissent certains cadres dirigeants, au début des années 1970, comme le fait Delfeil de Ton : "Ma parole, ils ne savent pas ce qu'ils font, ces ouvriers. [...] S'imaginent pas comme un patron c'est fragile. Ça prend des coups de sang facilement, un patron. Ça a pas la santé du manuel salarié. Ça mange trop de viande. Ça vit dans la plume. Ça mène une vie émolliente dans des appartements surchauffés. Les ouvriers ne connaissent pas leur bonheur. Ils croient que c'est marrant, les ouvriers, de se retrouver à Megève en plein mois de décembre, par un froid de canard et pas de neige ? [...] La vie des patrons, je voudrais que tout le monde puisse y goûter un peu, rien que pour savoir ce que c'est. ${ }^{6}$

4 Siné, Portée de chats, Paris, Jean-Jacques Pauvert, 1957.

5 On songe particulièrement au journal maoïste La Cause du peuple.

6 Charlie Hebdo, no 8, 11 janvier 1971. Une semaine plus tôt, Bernier faisait déjà référence à la séquestration d'un patron (Charlie Hebdo, $\mathrm{n}^{\circ}$ 7, 4 janvier 1971). 
Dans l'imagerie de Charlie, le capitaliste n'a guère évolué depuis l'époque de L'Assiette au beurre: il porte un chapeau haut-de-forme, fume un gros cigare, se caractérise par son arrogance, sa vénalité et son cynisme. À chaque échéance électorale, législative ou présidentielle, sa seule préoccupation semble être le maintien de sa fortune et de son patrimoine. C'est ainsi que, dans les dessins de Reiser et de Wolinski notamment, on le voit partir déposer son argent en Suisse par crainte d'une victoire de la gauche, et se réjouir bruyamment à chaque victoire de la droite. En 1973, Reiser dessine deux bourgeois ravis de la reconduction de la majorité : le premier remercie les Français «d'avoir bien voté " et le second se félicite : "Encore cinq ans à se remplir les poches. " ${ }^{7}$ L'année suivante, après la victoire présidentielle de Valéry Giscard d'Estaing, Reiser fait encore dire à un bourgeois rassuré : «On va continuer à péter dans la soie. $»^{8}$ Pour un journal écologiste comme Charlie Hebdo, le patronat industriel est en outre responsable de la dégradation de l'environnement, ainsi que l'affirme Gébé dans un dessin de couverture : "Pollution : les patrons puent de la gueule $"{ }^{9}$. Ce portrait finalement assez schématique, sinon caricatural, des patrons français peut s'expliquer par les origines sociales des membres de la rédaction : aucun collaborateur de Charlie Hebdo n'est issu de la grande bourgeoisie. Notons cependant que Wolinski est né dans une famille de la petite bourgeoisie commerçante ${ }^{10}$, ce qui le rend d'autant plus féroce envers ceux qui s'efforcent de copier la haute bourgeoisie.

Toutefois, en ces années 1970, celui qui incarne le mieux le pouvoir n'est pas, aux yeux de Charlie Hebdo, le patron, mais l'homme politique, et plus précisément celui qui appartient à la majorité et/ou au gouvernement, c'està-dire l'homme politique de droite, qu'il soit gaulliste ou libéral. Cavanna, Wolinski et quelques autres de leurs collègues l'ont souligné à plusieurs reprises, au risque de froisser d'autres membres de la rédaction voire certains lecteurs : Charlie Hebdo est un journal de gauche ${ }^{11}$, d'abord parce que c'est un journal qui rejette viscéralement la droite. "Tout ce qui est de droite est con " ${ }^{12}$, écrit même Cavanna. La droite représente en effet un ensemble de valeurs détestées par l'ensemble de l'équipe rédactionnelle : l'ordre social, le patriotisme exacerbé, la défense des puissants, la démagogie, l'attachement à la morale catholique... Ainsi, les représentants les plus éminents de cette élite politique sont constamment attaqués, moqués, et même ridiculisés. On songe ainsi à Michel Debré, auquel Wolinski inflige un entonnoir, en signe d'aliénation

7 Charlie Hebdo, n 122,19 mars 1973.

8 Charlie Hebdo, n 184, 27 mai 1974.

9 Charlie Hebdo, nº 62, 24 janvier 1972.

10 Ses parents tenaient une bonneterie.

11 "Nous sommes un journal de gauche, que nous le voulions ou non, pour vous, notre public " (Wolinski, in Charlie Hebdo, $\mathrm{n}^{\circ} 355,1^{\text {er }}$ septembre 1977).

12 Charlie Hebdo, no 119 bis, 20 février 1973. 
mentale ${ }^{13}$, entonnoir que nombre de lycéens reprennent lors des manifestations contre la loi Debré de réforme du service militaire, en mars et avril 1973. Charlie Hebdo plante également ses banderilles contre plusieurs ministres de l'Intérieur, incarnations du maintien de l'ordre social et de la répression policière. Raymond Marcellin, qui occupe la place Beauvau pendant les six années suivant Mai 68, est ainsi une des têtes de Turc favorites du journal, notamment lorsqu'il décide, en novembre 1970, d'interdire L'Hebdo Hara-Kiri après une couverture associant la mort du général de Gaulle au drame de SaintLaurent-du-Pont, au cours duquel, quelques jours plus tôt, 145 jeunes gens avaient trouvé la mort dans l'incendie d'une discothèque ${ }^{14}$. Cette censure le conduit, la semaine suivante, à changer de nom et à devenir Charlie Hebdo ${ }^{15}$. Huit jours plus tard, Raymond Marcellin obtient, bien malgré lui, le " Prix Bête et Méchant » de la part de la rédaction ${ }^{16}$.

Laffaire du "Bal tragique " rappelle aussi que le journal charge volontiers "l'élite de l'élite " politique, c'est-à-dire le chef de l'État, ce qui est assez naturel dans la " monarchie présidentielle " qu'est la $V^{e}$ République. Le général de Gaulle, qui démissionne moins de trois mois après le lancement de Hara-Kiri Hebdo, n'a guère le temps d'être attaqué par le journal. Il apparaît toutefois sous les traits d'un vieillard autoritaire, compassé et mégalomane, ami de Franco et de Salazar, sans être assimilé à Hitler comme le faisait Siné dans L'Enragé en 1968. Derrière son apparente bonhomie, doublée d'un esprit cultivé et raffiné, Georges Pompidou ne serait, selon les dessinateurs de Charlie Hebdo, qu'un vulgaire " patron de bistrot " - selon les termes de Reiser - ami du béton, du bitume, de l'ordre et des puissants, qu'il s'agisse de la bourgeoisie française ou de certains dictateurs étrangers. Pompidou représente enfin et surtout l'Anastasie des temps nouveaux : non seulement il a laissé son ministre de l'Intérieur interdire L'Hebdo Hara-Kiri, mais il aurait aussi demandé au parquet, en septembre 1972, d'ouvrir une information judiciaire contre X pour « offense au chef de l'État ", après la publication de l'album de Cabu, Les Aventures de Madame Pompidou, qui rassemble des strips parus pendant un an dans Charlie Hebdo. En juin 1973, le journal est condamné à une amende s'élevant à 5000 francs. Valéry Giscard d'Estaing, en revanche, avait promis pendant la campagne présidentielle de 1974 de ne pas jamais déposer plainte pour « offense au chef de

13 Charlie Hebdo, $n^{\circ}$ 105, 20 novembre 1972.

14 Il s'agit de la fameuse formule, imaginée par le professeur Choron : « Bal tragique à Colombey. 1 mort " (L'Hebdo Hara-Kiri, n 94, 16 novembre 1970).

15 Ce titre a pour origine un autre journal édité par la même maison, Charlie, journal de bandes dessinées dirigé par Wolinski. Le nom "Charlie " fait référence à un des personnages des Peanuts de Charles Schulz, Charlie Brown.

16 Charlie Hebdo, n 2, 30 novembre 1970. Le premier lauréat du «Prix Bête et Méchant " a été décerné en 1963 à un ami de l'équipe de Hara-Kiri, Jean-Christophe Averty, réalisateur de l'émission télévisée Les Raisins verts, à laquelle ont participé Cavanna et le professeur Choron. Le dernier lauréat sera un autre intime de la bande, Coluche, en 1978. 
l'État ». Il tient parole, malgré les multiples dessins et articles de Charlie Hebdo faisant de lui un monarque hautain et au modernisme affecté. Toutefois, son épouse obtient en 1980 la saisie du mensuel Hara-Kiri qui, dans un photomontage de couverture, la montre nue dans les bras d'un émir arabe ${ }^{17}$. En définitive, les élites politiques qui gouvernent la France jusqu'en 1981 apparaissent bien comme des cibles privilégiées du journal, tant et si bien que certains textes et certains dessins peuvent avoir un parfum poujadiste. "Un pour tous, tous pourris! » s'exclame ainsi Gébé en novembre 1971, dans une couverture consacrée au parti gaulliste, l'UDR ${ }^{18}$.

Dans une perspective à la fois anti-patronale et anti-gouvernementale, Charlie Hebdo s'oppose aussi à certains patrons de presse : Pierre Lazareff, patron de France-Soir, du Journal du dimanche, est ainsi accusé par Wolinski de diriger des journaux " pourris » et " dégueulasses ${ }^{19}$. Par ailleurs, on s'inquiète vivement des appétits médiatiques du " papivore » Robert Hersant, qui rachète successivement L'Aurore et Le Figaro, mais c'est le patron du Parisien libéré, Émilien Amaury, qui subit les attaques les plus violentes de la part de Charlie Hebdo. L'équipe du journal fustige précisément la ligne réactionnaire, populiste et même raciste qu'il imposerait à son quotidien. Après le décès accidentel d'Amaury, en 1977, la " une » de Charlie Hebdo, signée Gébé, est extrêmement violente : "Un salaud est mort $~^{20}$.

Néanmoins, Charlie Hebdo n'affirme jamais parler au nom du peuple ni ne se place dans la perspective d'un affrontement inévitable entre les classes populaires et les élites sociales. Ce refus de l'ouvriérisme s'exprime dans de nombreux dessins de Reiser, très violents à l'encontre d'un prolétariat dont il est lui-même issu ${ }^{21}$. Siné, fier de ses racines populaires, semble toutefois faire exception et s'en prendre exclusivement aux puissants, qu'il s'agisse des politiques, des patrons, des militaires, de policiers, des magistrats ou des hommes d'Église ${ }^{22}$. Outre Siné et Reiser, la plupart des membres de la rédaction ont des origines sociales plutôt modestes : Cavanna est ainsi le fils d'un maçon et d'une femme de ménage, et le professeur Choron, celui d'un cheminot et d'une

17 Hara-Kiri, n² 221, février 1980. On peut lire en « une »: "La France ne manquera pas de pétrole : Giscard offre sa femme aux émirs ".

18 Charlie Hebdo, n ${ }^{\circ}$ 54, 29 novembre 1971.

19 Hara-Kiri Hebdo, no 6, 10 mars 1969. Wolinski est alors également pigiste au Journal du dimanche...

20 Charlie Hebdo, no 321, 6 janvier 1977. Libération joue aussi la carte de la provocation en titrant : "Le cheval d'Amaury sort indemne d'un accident ».

21 Sa mère était femme de ménage et il est né de père inconnu.

22 Siné : "Reiser se moquait des cons qui partaient en vacances avec leur caravane. Je n'étais pas d'accord pour me foutre de la gueule de ces gens-là. Des capitalistes et des curés, oui, mais ça m'emmerdait qu'il traîne tout le monde dans la merde. Certains dessins dans Charlie Hebdo me choquaient. Nous n'avions pas la même définition de l'intolérable. " (cité par Jean-Marc Parisis, Reiser, Paris, Grasset, 1995). 
garde-barrière. En d'autres termes, ils ont grandi dans un monde étranger à celui des élites, ce qui a pu avoir des effets dans le regard qu'ils portent sur la culture savante. On note ainsi une relative distance à l'égard des élites intellectuelles, en témoigne ce silence sur les grands noms des sciences humaines de l'époque, de Barthes à Foucault et d'Althusser à Bourdieu ${ }^{23}$. L'émergence de nouveaux courants idéologiques entraîne souvent une forte méfiance politique : les " Nouveaux philosophes " apparaissent ainsi comme un groupement médiatique d'intellectuels de droite inavoués, et la « Nouvelle Droite», fondée par Alain de Benoist, ne serait qu'un rassemblement de racistes bon teint. Après la mort de Sartre, une des dernières figures de l'intellectuel " éditorial ", Cavanna confesse n'avoir rien compris à L'Existentialisme est un humanisme et affirme même : "Je ne suis pas un intellectuel ${ }^{24}$. Delfeil de Ton, seule figure du journal à avoir suivi des études supérieures, estime que ses collègues autodidactes "avaient peur des intellos ${ }^{25}$. Cependant, le même Delfeil, dans Charlie Hebdo, condamne avec force le « racisme culturel » dont feraient preuve certains " intellectuels et journalistes assis sur [leurs] piles de bouquins de la NRF " et qu'il accuse de "s'aveugler sur les vraies lectures populaires ${ }^{26}$. Le journal se montre aussi particulièrement acerbe contre les représentants d'une culture "bourgeoise ", qu'incarneraient les écrivains membres de l'Académie Goncourt, mais aussi, de façon plus surprenante, certains cinéastes vieillissants de la défunte Nouvelle Vague, à l'instar de Claude Chabrol et François Truffaut : Delfeil juge le premier comme le " meilleur représentant " du régime pompidolien et il accuse le second d'avoir versé dans le conformisme absolu, notamment dans La Nuit américaine. Finalement, à travers l'exemple de la culture, Charlie Hebdo n'apparaît pas seulement comme un journal opposé aux élites " traditionnelles ", mais aussi comme un journal représentatif d'une certaine contre-culture, et partant d'une contre-élite.

\section{Charlie Hebdo, figure de proue d'une « contre-élite »?}

À plusieurs titres, Charlie Hebdo peut être défini comme l'un des principaux journaux français de la contre-culture, d'abord parce qu'il porte une parole contestant l'ordre établi, et parce qu'il se situe lui-même en marge des grands circuits commerciaux, comme il l'a toujours revendiqué, et ce dès la création de Hara-Kiri en 1960 : Cavanna n'entendait pas, avec ce mensuel, s'adresser à l'ensemble des Français, mais à cette minorité de "lecteurs

23 Bourdieu a néanmoins un point commun avec Charlie Hebdo : il soutient la candidature de Coluche à l'élection présidentielle de 1981.

24 Charlie Hebdo, no 493, 23 avril 1980.

25 Entretien du 11 avril 2002.

26 Charlie Hebdo, n 140, 24 juillet 1973. 
exigeants sur la qualité ${ }^{27}$. L'ambition est identique pour Charlie Hebdo : il s'agit de "faire le plein des affamés "d'autre chose" et mordre peu à peu sur la frange récupérable des résignés faute de mieux à la connerie tonitruante. ${ }^{28}$ En outre, soucieux de préserver sa totale indépendance d'esprit, Charlie Hebdo ne reçoit aucun financement de la publicité. Son inscription dans la contreculture se vérifie également par le mode d'expression graphique de certains de ses dessinateurs, à commencer par Willem, de nationalité néerlandaise, très proche du mouvement provo dans les années 1960, et grand admirateur de Robert Crumb, "pape » de la contre-culture américaine. Willem tient d'ailleurs dans Charlie Hebdo une revue de presse particulièrement originale en ce sens qu' elle est consacrée de façon quasi-exclusive à la presse dite underground, qu'elle soit américaine, européenne, ou plus spécifiquement française, avec des journaux comme Actuel de Jean-François Bizot, puis dans une moindre mesure, à partir de 1973, Libération, enfant de Mai 68 et de la presse gauchiste, et dont Charlie Hebdo soutient activement la fondation. Dans ce réseau « contre-culturel » dont Charlie Hebdo serait l'un des nœuds, on peut également inclure certaines maisons d'édition comme celles de Pauvert, qui, dès les années 1960, a publié Cavanna, Wolinski, ainsi que deux journaux de Siné, Siné Massacre et L'Enragé, ou encore de Régine Desforges et d'Éric Losfeld. Ce réseau compte également un mouvement artistique né en 1962 et qui se définit lui-même comme un "anti-mouvement ": Panique, fondé par Fernando Arrabal, Alejandro Jodorowsky et Roland Topor, collaborateur de Hara-Kiri entre 1961 et $1965^{29}$. À travers cet exemple se pose la question de l'appartenance de Charlie Hebdo à une certaine avant-garde culturelle, comme le revendique Gébé : «L'histoire contemporaine retiendra peut-être la filiation avec les surréalistes et Dada. Nous sommes de la lignée de ces gens-là » ${ }^{30}$. Le même Gébé a imaginé, au début des années 70, "L'An 01 ": une société utopique fondée sur le partage du travail, la liberté sexuelle, la vie en communauté : en d'autres termes, des valeurs opposées à la culture dominante. Sa bande dessinée fait l'objet en 1972 d'une adaptation cinématographique à laquelle participent de nombreux collaborateurs de Charlie Hebdo. Le film L’An 01, réalisé par Jacques Doillon, fait incontestablement figure de film underground, aussi bien par son mode de financement que par le discours qu'il délivre. Enfin, Charlie Hebdo est un indéfectible soutien au théâtre " alternatif ", c'est-à-dire en rupture avec les règles académiques du spectacle vivant, notamment le Grand Magic Circus de Jérôme Savary, que les articles élogieux

27 Cavanna, Bête et méchant, op. cit.

28 Cavanna, Les Années Charlie, Paris, Hoëbeke, 2004.

29 Jacques Sternberg, auteur de plusieurs textes pour Hara-Kiri et Charlie Hebdo, a également participé au mouvement Panique.

30 Le Monde, 7 février 1999. Cf. aussi Mazurier Stéphane, "Hara-Kiri, un journal d'avantgarde ", Histoires littéraires, n 26, avril-mai-juin 2006, p. 5-28. 
de Delfeil de Ton contribuent à faire connaître, mais aussi le café-théâtre, notamment le Café de la Gare, fondé par Coluche et Romain Bouteille en 1969 , tous deux très proches de la "bande à Charlie " ${ }^{31}$.

À ce propos, le succès grandissant des comédiens du Café de la Gare, puis de ceux du Splendid, fondé en 1974 par Thierry Lhermitte, Gerard Jugnot, Christian Clavier et Marie-Anne Chazel, révèle une certaine diffusion, voire une banalisation de l'esprit "bête et méchant ", c'est-à-dire cet humour noir, provocateur, parfois scatologique, véhiculé par Hara-Kiri et Charlie Hebdo. Cela explique que, notamment à partir de la seconde moitié des années 1970, les membres de la rédaction ont pu finalement s'intégrer à des réseaux plus traditionnels des élites médiatiques et culturelles. Ainsi, non seulement Cavanna, Wolinski, Cabu et Reiser fréquentent-ils régulièrement les plateaux de télévision et les studios de radio, ainsi les émissions Apostrophes sur Antenne 2 ou Radioscopie sur France-Inter, mais aussi nouent des contacts avec quelques figures de l'élite intellectuelle : Cavanna se lie d'amitié avec l'écrivain et journaliste Bertrand Poirot-Delpech, Reiser avec Georges Perec, ainsi qu'avec l'architecte suisse Mario Botta. Parallèlement à l'effondrement des ventes de Charlie Hebdo, les livres de Cavanna et de Reiser sont de gros succès de librairie. La deuxième partie de l'autobiographie de Cavanna, Les Russkoffs, obtient même le Prix Interallié 1979, signe d'une incontestable consécration élitaire ${ }^{32}$. Par ailleurs, à partir de la seconde moitié de la décennie soixante-dix, nombre d'entre eux travaillent, parallèlement à Charlie Hebdo, pour de grands titres de la presse « sérieuse ». Pendant l'été 1978, à la demande de Bruno Frappat, Reiser publie en effet dans Le Monde une série intitulée La Famille Oboulot en vacances. Cette expérience fait cependant long feu en raison de l'hostilité d'une partie des lecteurs et de la rédaction, qui ne voit en Reiser qu'un " pornographe ". Cette hostilité tendrait d'ailleurs à montrer les limites de cette intégration aux élites et de cette "institutionnalisation " de Charlie Hebdo par la collaboration d'un de ses dessinateurs majeurs au quotidien français « de référence ». Trois ans plus tard, Reiser rejoint néanmoins Le Nouvel Observateur, magazine emblématique de l'élite intellectuelle de gauche, à la demande de Delfeil de Ton qui, en 1975, a lui-même quitté le journal de Cavanna pour celui de Jean Daniel. Quant à Wolinski, il multiplie les collaborations, que ce soit au Journal du dimanche de Pierre Lazareff, dès la fin des années 1960, ou bien à L'Humanité, dont il signe, à partir de 1977, le dessin de couverture. Dans ce dernier cas, on voit qu'un membre historique de Charlie Hebdo peut ainsi se lier à une certaine contre-élite politique, en l'occurrence l'un des plus puissants mouvements de l'opposition au pouvoir gaullo-giscardien, le Parti communiste français. La

31 D'après le titre d'un ouvrage de Jean Egen, La Bande à Charlie, Paris, Stock, 1975.

32 Cavanna, Les Russkoffs, Paris, Belfond, 1979. Au micro de France-Inter, Jacques Chancel demande plaisamment à Cavanna si sa prochaine étape n'est pas l'élection à l'Académie française. «Pouah!", répond le rédacteur en chef de Charlie Hebdo (Radioscopie, 27 décembre 1979). 
démarche de Wolinski, très critiquée par ses collègues car remettant en cause le principe d'indépendance d'esprit du journal, est certes isolée, mais on ne peut nier les passerelles qui existent entre certains membres de la rédaction et certains responsables politiques de l'opposition de gauche. C'est ainsi que Reiser connaît les dirigeants de L'Humanité, Roland Leroy et Pierre Andrieu, depuis le milieu des années 1970, et que c'est même lui qui les présente à Wolinski. Quant à Cabu et son épouse Isabelle, ils sont un temps proches du Parti socialiste unifié de Michel Rocard, incarnation d'une "nouvelle gauche ", plus moderne que la vieille SFIO de Guy Mollet.

Lorsque la " contre-élite " politique de gauche parvient au pouvoir au printemps 1981 et devient en quelque sorte l'élite politique "officielle », la position de Charlie Hebdo s'en trouve malaisée. En effet, ce journal, de tradition contestataire, approuve la plupart des premières mesures du gouvernement socialiste : l'abolition de la peine de mort, l'augmentation des prestations sociales, la baisse du temps de travail, les nationalisations... Doit-il poursuivre néanmoins son attitude d'opposition au pouvoir, au risque de sombrer dans le nihilisme politique ? Ou bien doit-il applaudir les réformes de gauche, au risque de devenir un simple compagnon de route de la nouvelle élite politique ? Gébé rend compte de cette situation schizophrénique dans un entretien accordé en 2002 : "On a été victimes de l'arrivée de la gauche parce que la gauche a créé à l'époque un tel espoir que beaucoup de gens nous ont dit : "Ben, ça y est : votre combat est fini." Par contre, les lecteurs "durs" disaient : "Ça y est : la gauche est arrivée, donc on vous a limé les dents !" Alors les uns pensaient qu'on émettait des critiques qui étaient hors de propos, dépassées et les autres pensaient qu'on n'avait plus du tout de virulence. Et c'est impossible de s'en sortir et les ventes baissaient. "33 $^{33}$

Ainsi, même si le journal meurt en janvier 1982 pour des raisons financières, on peut donc également invoquer des facteurs politiques à cette disparition, puisqu'un hebdomadaire par nature irrévérencieux, marginal, se surprend désormais à soutenir la classe dirigeante et donc à se situer du côté des nouvelles élites. Dans le dernier numéro de Charlie Hebdo, le dessinateur Carali écrit, avec humour, mais aussi avec justesse, que le journal a été, d'une certaine façon, « assassiné par le socialisme " ${ }^{34}$.

En définitive, Charlie Hebdo n'est certes pas un journal élitiste ni même élitaire, mais il n'est pas non plus un journal populiste, dressant un prolétariat nécessairement vertueux contre des élites forcément corrompues. Il entend

33 Entretien du 6 novembre 2002. Cabu est du même avis : selon lui, l'arrivée de la gauche au pouvoir "a précipité la chute de Charlie " (Cabu, politiquement incorrect, France 5, 20 septembre 2006).

34 L'Hebdo Hara-Kiri, no 23 (= Charlie Hebdo, n 580), 23 décembre 1981. 
plutôt incarner une avant-garde, être, comme le disait Gébé, un " journal de pointe, le leader d'opinion dans un certain mode de pensée $»^{35}$, que l'on peut grossièrement résumer comme la "pensée 68 ", c'est-à-dire une pensée libertaire, féministe, écologiste, anti-consumériste. Enfin, si ce journal n’a pas pour ambition d'être admis dans les cercles du pouvoir culturel et médiatique et qu'il affiche même parfois sa fierté de ne pas y être convié, il s'y est progressivement et partiellement intégré à partir de la seconde moitié des années 1970. Toutefois, les rapports que le journal de Choron et de Cavanna entretient avec ces élites sont infiniment plus distants que ceux tissés par Philippe Val, lorsque ce dernier dirige la deuxième série de Charlie Hebdo, entre 1992 et 2009. Val n'a de cesse, surtout à partir de 1999-2000, de vouloir s'agréger à une sorte d'aristocratie éditoriale et médiatique. Il devient, en effet, un proche de Laurent Joffrin, alternativement directeur de Libération et du Nouvel Observateur, de BernardHenri Lévy, qui l'a fortement soutenu durant l'affaire de caricatures de Mahomet en 2006-200736, ou encore de Jean-Luc Hees. Quand, en mai 2009, ce dernier est nommé président de Radio-France par le Président Sarkozy, il choisit son ami Philippe Val pour la direction de France-Inter, suivant ainsi les recommandations de l'Élysée, et plus précisément de Carla Bruni-Sarkozy, intime du directeur de Charlie Hebdo ${ }^{37}$. On éprouve quelques difficultés à imaginer le même scénario, quarante ans plus tôt, soit le général de Gaulle nommant le professeur Choron à la tête de l'ORTF. Dans son récit autobiographique, Lune de miel, Cavanna écrit à ce propos que tout s'est passé comme si le «fabuleux journal [de Reiser] n'avait existé que pour assurer la promotion sociale d'un ambitieux »: "Val a quitté l'hebdo pour des destinées plus hautes et plus prometteuses. Le passage au journalisme n'aura été pour lui qu'un marchepied vers des conquêtes sans cesse plus brillantes. ${ }^{38}$ Journal né de la volonté de s'attaquer avec humour à toutes les élites, Charlie Hebdo semble ainsi devenu aujourd'hui, une sorte de strapontin pour accéder à ce monde : un temps chroniqueuse pour le journal, Caroline Fourest a reçu en 2006 le Prix de l'Assemblée nationale des mains de Jean-Louis Debré.

35 Entretien du 6 novembre 2002.

36 Lors de cette affaire, Charlie Hebdo a reçu le soutien de l'ensemble des élites politiques, notamment François Bayrou, Nicolas Sarkozy et François Hollande. On a retrouvé ce même soutien après l'incendie criminel des locaux de Charlie Hebdo, consécutif au numéro spécial "Charia Hebdo " (n 1011, 2 novembre 2011). Le ministre de l'Intérieur Claude Guéant a même dénoncé un « attentat » et apporté le soutien du gouvernement à l'équipe de Charlie Hebdo.

37 Le Nouvel Observateur, 21 mai 2009.

38 François Cavanna, Lune de miel, Paris, Gallimard, 2011, p. 271. 
Cinq ans plus tard, le président du Sénat, Jean-Pierre Bel, a nommé Bernard Maris, alias " Oncle Bernard » de Charlie Hebdo, au conseil général de la Banque de France ${ }^{39}$.

\author{
Stéphane Mazurier \\ Lycée Camille-Jullian, Bordeaux \\ mazurier.stephane@orange.fr
}

\begin{abstract}
Résumé
Journal emblématique des " années 68 ", Charlie Hebdo a notamment exercé sa verve satirique envers certaines élites de la société française : les industriels, les magnats de la presse, mais surtout les responsables politiques de la droite gaullo-giscardienne. Ce discours anti-élitaire ne dévie cependant pas vers le populisme, ni même l'ouvriérisme. Les membres de la rédaction sont majoritairement issus des classes populaires, et portent un regard très distancié sur les élites intellectuelles classiques. En revanche, ils s'inscrivent parfaitement dans la « contre-culture » des années soixante-dix et s'agrègent ainsi à une espèce de "contre-élite ". Enfin, la victoire de la gauche en 1981 a pour effet de placer Charlie Hebdo dans une position délicate de soutien aux nouvelles élites politiques au pouvoir.
\end{abstract}

\title{
Mots-clés
}

Presse satirique, France, satire politique française, caricatures et dessins humoristiques, élites.

\begin{abstract}
Emblematic newspaper of "years 68", Charlie Hebdo practiced in particular its satirical liveliness towards French society elites: industrialists, press barons, but especially gaullist and giscardian Right political leaders. However, its counter-elites speech does not move away towards populism. The editorial board is made up popular classes, it takes a distant look at the traditional intellectual elites. Yet, it fits perfectly the "counter-culture" of the Seventies and thus incorporates itself with a sort of "Counter-elite". At last, the victory of the Left in 1981 places Charlie Hebdo in a delicate support for the new political elites in power.
\end{abstract}

\section{Keywords}

Satirical newspapers, France, french political satire, caricatures and cartoons, elites.

39 Bernard Maris fut, jusqu'en 2009, l'un des quatre actionnaires de Charlie Hebdo. Il a été marié à la journaliste Sylvie Genevoix, fille de l'académicien Maurice Genevoix et membre du Conseil supérieur de l'audiovisuel de 2005 à 2011. Quelques mois avant sa mort, en 1983, Reiser avait lui-même épousé une journaliste, Michèle Jouhaud-Castro, qui devait devenir membre du CSA, une vingtaine d'années plus tard. 